\title{
Emotional and non-emotional facial behaviour in patients with unilateral brain damage
}

\author{
JOAN C BOROD, ${ }^{*} \dagger$ ELISSA KOFF, $\ddagger$ MARJORIE PERLMAN LORCH,§ \\ MARJORIE NICHOLAS,§ JOAN WELKOWITZ\|
}

From the Departments of Psychology, Queens College, and Neurology, Mount Sinai Medical School,* The City University of New York, Department of Psychiatry, $\dagger$ New York University Medical School, Department of Psychology, $\ddagger$ Wellesley College, Aphasia Research Center, $\S$ Boston VA Medical Center, and Department of Neurology, Boston University Medical School, Department of Psychology, \|N New York University, USA

SUMMARY Aspects of emotional facial expression (responsivity, appropriateness, intensity) were examined in brain-damaged adults with right or left hemisphere cerebrovascular lesions and in normal controls. Subjects were videotaped during experimental procedures designed to elicit emotional facial expression and non-emotional facial movement (paralysis, mobility, praxis). On tasks of emotional facial expression, patients with right hemisphere pathology were less responsive and less appropriate than patients with left hemisphere pathology or normal controls. These results corroborate other research findings that the right cerebral hemisphere is dominant for the expression of facial emotion. Both brain-damaged groups had substantial facial paralysis and impairment in muscular mobility on the hemiface contralateral to site of lesion, and the left brain-damaged group had bucco-facial apraxia. Performance measures of emotional expression and non-emotional movement were uncorrelated, suggesting a dissociation between these two systems of facial behaviour.

The neuropsychological mechanisms involved in the facial expression of emotion have been the subject of a number of recent investigations. ${ }^{12}$ While the majority of these studies have used normal adult subjects, several have examined facial behaviours in braindamaged populations. Studies in normal subjects have documented that the left side of the face (or "hemiface"), presumably controlled by the right cerebral hemisphere, ${ }^{3}$ moves more extensively ${ }^{4-6}$ and appears more intense ${ }^{7-10}$ during emotional expression than does the right hemiface (but see also ${ }^{11}$ ). Studies of facial emotional expression in brain-damaged patients, ${ }^{12-15}$ also have suggested a special role for the right hemisphere in emotional expression. In these studies, right brain-damaged patients, relative to left brain-damaged patients and normal controls, are more impaired in the production of facial emotional expressions.

The main purpose of this study was to elucidate

Address for reprint requests: Dr Joan C Borod, Department of Psychology, Queens College, CUNY Flushing, NY 11367, USA.

Received 21 August 1987 and in revised form 16 December 1987. Accepted 23 December 1987 aspects of facial emotional expression that have previously been associated with unilateral brain damage. Facial expressions from an earlier study ${ }^{13}$ were reexamined, and three aspects of facial behaviour were analysed: appropriateness, intensity, and responsivity. These parameters were selected because of observations in the clinical literature that right hemisphere pathology is associated with inappropriate affect, ${ }^{16}$ flattened affect, ${ }^{17}$ and low levels of arousal. ${ }^{18}$ To our knowledge, there has been no attempt to examine systematically these various aspects of facial expression in a brain-damaged population. As in our previous work with normals, ${ }^{5}$ we employed measures of expression appropriateness to address the issue of inappropriate affect, measures of intensity to address the issue of "flat affect," and measures of responsivity to address the issue of decreased arousal. In addition to providing a more qualitative analysis of facial expression deficits, the approach adopted for this study permits an examination of the interrelationships among these various measures. It would be important from a theoretical perspective to ascertain whether impairments in responsivity, appropriateness, and intensity are independent and separate from each other or whether they reflect a common 
underlying deficit in facial emotional expression.

A second purpose of this study was to examine how facial movement relates to the expression of facial emotion. While outcomes of facial emotional expression studies typically have been ascribed to central (that is, cerebral) processing mechanisms, the possibility that these findings could reflect other characteristics of the face has to be considered. Studies of normal subjects have reported that asymmetries in emotional facial expression are not correlated with asymmetries in non-emotional muscular mobility ${ }^{19}$ or in hemiface size. ${ }^{20}$ In brain-damaged patients, it remains to be determined whether deficits in facial motor behaviour, such as facial paralysis, might contribute to impairment in facial emotional expression. Such deficits could affect the brain-damaged patient's ability to execute tasks of facial emotion, and could influence the examiner's judgements about behaviours associated with such tasks. For example, the face of a patient with a dense central facial paralysis may appear so distorted or unusual that any emotional expression would be perceived as inappropriate. Also, a patient with restricted mobility of the facial musculature might produce emotional expressions of limited intensity, creating the impression of flattened affect. Finally, a patient with bucco-facial apraxia may be unable to respond to a command to use his or her facial muscles to express an emotion.

In the current study, measures of facial emotion (under posed and spontaneous conditions) were correlated with measures of related facial movements. Posed expressions were studied in a manner designed to parallel directed clinical examination of affect; spontaneous expressions were elicited to provide a naturalistic analogue for "clinical observations" of patient behaviour. Aspects of facial movement considered in this report were non-emotional hemiface mobility ${ }^{21}$ facial paralysis, ${ }^{22}$ and bucco-facial apraxia. $^{23}$

\section{Methods}

Subjects

Background data Subjects were right-handed (by self- or family-report) adult males with unilateral right braindamage (RBD) $(\mathrm{N}=12)$ or left brain-damage (LBD) $(\mathrm{N}=$ 15); 16 normal males (NC) served as controls. The three subject groups did not differ significantly on demographic variables, with an overall mean age of 57 years $(S D=8 \cdot 1)$, 13 years of education (SD $=2 \cdot 8)$, and an occupational level of $3.9(\mathrm{SD}=1.5)$ as measured by the Hollingshead-Redlich seven-point scale. ${ }^{24}$

Neurological data Patients were tested at least one month after onset of illness (median $=9$ months) and only were included in this study if their lesions were the result of a cerebrovascular accident and if there was a negative history of psychiatric disorder, psychotropic drug treatment, or secondary neurological disorder (such as epilepsy, dementia).
Evidence of the unilateral and focal nature of the lesion was confirmed by CT in all but two cases, where clinical data from neurological examination were used. Fourteen $(63 \%)$ of the patients had lesions restricted to the cortex and/or subcortical white matter, while the remainder had cortical lesions which extended to subcortical grey matter structures (for example, basal ganglia, thalamus). The patient groups were similar with regard to the distributions of their lesions. The percentages of patients with lesions in a particular region, for RBDs and LBDs respectively were: frontal- $58 \%$, $60 \%$; parietal-92\%, 67\%; temporal-58\%, 67\%; occipital-17\%, 7\%; subcortical white matter-83\%, 60\%; and subcortical grey structures-50\%, 27\%. Using the Fisher exact probability test to examine the frequency of RBD and LBD patients with or without a lesion in each region, none of the comparisons were significant. (Since the sensitivity of CT scanning is limited, other lesions that were not detectable by this method may have been present in these patients.) At the time of testing, the majority of each patient group had sensory-motor deficits on the side contralateral to their lesion. These deficits were hemiplegia ( $58 \%$ of RBDs, $60 \%$ of LBDs); central facial paralysis $(75 \%$ of RBDs, $80 \%$ of LBDs); and visual field loss $(58 \%$ of RBDs, $47 \%$ of LBDs). Fisher tests were conducted, and, again, there were no significant group differences. Although all of the LBDs were aphasic (3 Broca's, 2 Mixed Nonfluents, 2 Globals, 2 Conductions, 4 Wernicke's, 2 Unclassifieds), all had adequate auditory comprehension (mean Boston Diagnostic Aphasia Examination ${ }^{23}$ z-score = $-0.88, \mathrm{SD}=0.70$ ) and cognitive functioning (mean WAIS Performance $\mathrm{IQ}^{25}=94, \mathrm{SD}=16$ ) to perform the experimental tasks. All subjects had intact basic visual perception, as assessed by the ability to correctly describe two slides, that is, a kindergarten classroom scene $\mathbf{3}^{34}$ and a Thematic Apperception Test card.

Dimensions of non-emotional facial movement In order to determine whether the brain-damaged groups were equivalent on facial movement, videotapes of subjects' faces were rated for facial paralysis (resting face) ${ }^{26}$ and hemiface mobility. ${ }^{21}$ To control for the effect of central facial paralysis, and since the lower face predominantly receives contralateral innervation while the upper face receives bilateral innervation, ${ }^{32-29}$ the upper and the lower face were assessed separately. Bucco-facial apraxia also was evaluated because of concern that it might influence the performance of LBDs on tasks of facial emotional expression.

Facial paralysis For upper face paralysis, pattern of wrinkles in the forehead, position of eyebrow, and size of eyelid were assessed; for lower face paralysis, depth of the nasolabial fold, position of the corner of the mouth, and direction to which the middle of the mouth is pointing were assessed, according to standard procedures. ${ }^{30-32}$ For each of these parameters, asymmetry was rated as 1 (more paralysis on left), 2 (no asymmetry), or 3 (more paralysis on right). Faces were rated by two independent judges, with $84 \%$ complete agreement on a subsample of 67 observations; the mean of the two ratings was used in data analysis. Each subject received one score for the upper face ( $\overline{\mathrm{x}}$ of the three parameters) and one for the lower face ( $\bar{x}$ of the three parameters). One-way analyses of variance (ANOVAs) on the asymmetry ratings revealed no difference among the three subject groups for the upper face. For the lower face 
Table 1 Mean scores (and standard deviations) for measures of non-emotional facial movements, by subject group

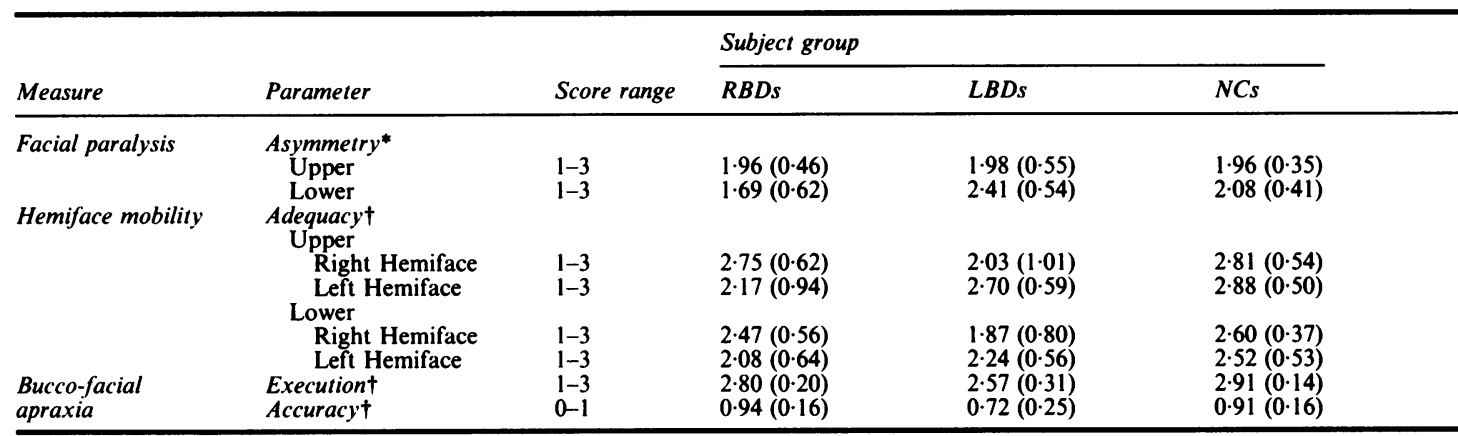

*From left-sided (1) to right-sided (3), with symmetry (2) as the midpoint.

+From impaired (low score) to unimpaired (high).

( $F=6.75 ; \mathrm{df}=2.40 ; \mathrm{p}<0.01)$, RBDs showed left-sided paralysis, LBDs right-sided paralysis, and NCs no paralysis/asymmetry. Means for each subject group are displayed in table 1 .

Facial mobility Subjects were instructed to make four unilateral facial movements, one involving eye (upper face) and three involving mouth (lower face) ${ }^{21}$ Each movement was made five times in succession on each side of the face. For each of these movements, each hemiface was rated as 1 (inadequate), 2 (partially adequate), or 3 (adequate). Two independent raters were in agreement for $83 \%$ of a subsample of 80 observations, and the mean of the two ratings used for data analysis. Each subject received one score for the upper face and one for the lower face ( $\bar{x}$ of the three movements). Two-way ANOVAs were conducted on the adequacy ratings to examine the effects of Group and Hemiface, separately for the upper and lower face. (See table 1 for group means.) While there was no main effect of Group for the upper face, there was an effect of Group for the lower face (F = 3.81; df $=2.40 ; \mathrm{p}<0.05)$, wherein LBDs $(\overline{\mathrm{x}}=2.06)$ and RBDs $(\overline{\mathrm{x}}$ $=2.28)$ were more impaired than NCs $(\overline{\mathrm{x}}=2.56)$. There was a Group by hemiface interaction for both the upper $(F$ $=7.56 ; \mathrm{df}=2.40 ; \mathrm{p}<0.005)$ and the lower $(\mathrm{F}=5.02 ; \mathrm{df}$ $=2.40 ; p<0.01)$ faces. For both the upper and the lower face, the left Hemiface was less impaired than the right among LBDs while the right was less impaired than the left among RBDs; there were no differences for NCs.

Facial apraxia Subjects were requested to perform four movements: suck through a straw, blow out a match, sniff a flower, and lick an envelope. ${ }^{33}$ Each movement was rated for execution as 1 (poorly or clumsily executed), 2 (slightly clumsy), or 3 (well-executed), and for accuracy as $\mathbf{0}$ (inaccurate) or 1 (accurate). To receive a score of 1 on accuracy, shape, movement (that is, direction), and location had to be correct. Since interrater complete agreement was high for both execution $(84 \%)$ and accuracy $(100 \%)$ on a subsample of 80 observations, the mean of the two ratings was used for each movement. Subjects received separate apraxia scores $(\bar{x}$ of the four movements) for execution and accuracy. Separate one-way ANOVAs examined the effect of Group. There was a significant main effect of Group for both execution ( $F$ $=8.73 ; \mathrm{df} 2.40 ; \mathrm{p}<0.001)$ and accuracy $(\mathrm{F}=5.10 ; \mathrm{df}=$ $2.40 ; p<0.01)$. In both cases, LBDs were more impaired than RBDs or NCs. (See table 1 for group means.)

\section{Experimental procedures}

Subjects were videotaped, as part of a larger study, ${ }^{12} 13$ while producing positive (that is, pleasant) and negative (that is, unpleasant) facial emotional expressions, under spontaneous and posed conditions. Valence categories (positive, negative) were based on findings from an earlier study of normal adults ${ }^{519}$ in which facial expressions were rated by trained judges for the degree of pleasant versus unpleasant affect.

For the spontaneous condition, slides designed to elicit expressions of positive and negative emotion ${ }^{34}$ were presented to the subject on a Singer Caramate projector $\cong$ There were 10 positive and six negative slides. Positive slides were selected from each of four categories; pleasant scenes scenic views, sexual material, and familiar people. Negative slides were selected from each of three categories: disgustingo materials, sad scenes, and unusual photographs. Each cate gory was represented by two slides, except for familiar peo ple where there were four slides. For example, one positive slide showed a little baby picking flowers, and one negative slide depicted a surgical procedure. Subjects were seated facing a one-way mirror, behind which a videocamera recorded their facial expressions. Each of the slides was presented for 20 seconds; after the first 6 seconds, the subject was requested to describe his feelings about and reactions to the slide.

For the posed condition, subjects were requested to deliberately pose three positive (happiness, pleasant surprise, sexual arousal) and five negative (sadness, anger, fear, disgust, confusion) emotional expressions to oral command, such as "Look happy." The cue for the subject to actually pose the expression was "Ready, go." Expressions were posed two times, once in response to a verbal command and once in response to visual imitation. Both auditory verbal (oral vignettes) and visual nonverbal (photographs of prototypical expressions) ${ }^{35}$ instructions were used to avoid selectively priming one or the other hemisphere through the input modality. To keep the spontaneous and posed conditions as parallel as possible, no encouragement was given to maximise or intensify responses.

Two judges viewed the videotapes and rated each facial expression for responsivity, appropriateness, and intensity. For the spontaneous condition, the first complete facial expression following the onset of each slide was rated. For the 
posed condition, the expression following "Ready, go" was rated. Half of the videotapes (containing both emotional and non-emotional movements) were rated under normal viewing conditions and half were rated under mirror-image conditions; this was done to control for perceiver bias. ${ }^{37}$ Responsivity ratings were made on a two-point scale $(0=$ no, $1=$ yes) based on rater consensus as to whether an expression had occurred or not. If an expression occurred, it was then rated for appropriateness and intensity. Appropriateness ratings were made on a two-point scale $(0=$ inappropriate, $1=$ appropriate). For a response to be scored as appropriate, consensus of the two raters was necessary, and the facial expression had to be appropriate to the stimulus slide or command. For the spontaneous condition, the raters were familiarised with each of the 16 stimulus slides. For the posed condition, the raters were familiarised with photographic exemplars of prototypical facial expressions of happiness, surprise, sadness, anger, fear, disgust, ${ }^{35} 36$ sexual arousal, and confusion. ${ }^{45}$ Overall, there was $98 \%$ complete agreement for the appropriateness ratings. Intensity ratings were made on a 7-point scale from "minimal" to "maximal" intensity. Since interrater agreement, determined on a subsample of observations, was high (Pearson's $r=+0.74$ ), a mean score of the two ratings was used for this variable. The categorisation of emotional expressions as positive or negative was based on a priori classification schemes ${ }^{34} 36$ and on post hoc analyses. (Using Chi Square Tests, score distributions for all individual expressions within a valence category were compared to each other. Responsivity, appropriateness, and intensity were examined in both posed and spontaneous conditions, separately by subject group. Among the 468 Chi Square Tests conducted, only $4 \%$ were significant and no patterns emerged). For each of the three dependent variables (responsivity, appropriateness, intensity), subjects received a separate mean score for posed positive (the $\bar{x}$ of the six expressions [three verbal, three visual]), posed negative (the $\bar{x}$ of the 10 expression [five verbal, five visual]), spontaneous positive (the $\bar{x}$ of the 10 slides) and spontaneous negative ( $\overline{\mathrm{x}}$ of six slides) emotions.

\section{Results}

\section{Group comparisons for emotional facial expression}

A series of repeated measures analyses of variance (ANOVAs) were conducted on the emotional facial expression variables, that is, responsivity, appropriateness, and intensity. For each variable, the effects of Group (RBD, LBD, NC), Condition (Posed, Spontaneous), and Valence (Positive, Negative) were examined in a $3 \times 2 \times 2$ design. Only significant effects are reported, and the Newman-Keuls multiple comparison procedure ${ }^{38}$ was used for post hoc tests.

Responsivity The three-way ANOVA conducted on the responsivity scores yielded a significant main effect of Group ( $F=3.64$; df $=2.40 ; p<0.05)$. Using post hoc tests, the RBDs were significantly ( $p$ $<0.05$ ) less responsive than either the LBDs or NCs. (See table 2 for group means.) There also was a main effect for Condition $(F=69.62$; df $=1.40 ; p<$ 0.001 ), such that subjects were more responsive in the posed $(\bar{x}=0.89)$ than in the spontaneous $(\bar{x}=0.48)$ condition. Finally, there was a Condition by Valence interaction $(F=5.42 ; \mathrm{df}=2.40 ; \mathrm{p}<0.05)$. Using post hoc tests to examine condition differences within each valance category, for the positive category, subjects were significantly more responsive in the posed $(\overline{\mathrm{x}}=0.90)$ than the spontaneous $(\overline{\mathrm{x}}=0.43)$ condition; for the negative category, again, subjects were significantly more responsive in the posed $(\bar{x}=0.87)$ than the spontaneous $(\bar{x}=0.52)$ condition. Using post hoc tests to examine valence differences within each condition, in the spontaneous condition, subjects were significantly more responsive on negative $(\overline{\mathbf{x}}$ $=0.52)$ than positive $(\bar{x}=0.43)$ expressions; there were no differences for the posed condition.

Appropriateness The three-way ANOVA conducted on the appropriateness scores yielded a significant main effect of Group ( $F=4.05 ; \mathrm{df}=2.34 ; p<0.05)$. Using post hoc tests, the RBDs were significantly (p $<0.05$ ) more impaired than the NCs. (See table 2 for group means.) There also was a significant interaction between Group and Valence $(F=3.46$; $\mathrm{df}=2.34$; $p$ $<0.05)$. Using post hoc tests to examine group differences within each valence category, $\operatorname{RBDs}(\overline{\mathbf{x}}=$ $0.78)$ were significantly $(p<0.05)$ less appropriate than LBDs $(\overline{\mathrm{x}}=0.91)$ or NCs $(\overline{\mathrm{x}}=0.95)$ for positive emotions; the groups did not differ for negative emotions (RBDs $=0.88$, LBDs $=0.84, \mathrm{NCs}=0.93$ ). Using post hoc tests to examine valence differences within each group, RBDs had significantly more difficulty with positive $(\overline{\mathrm{x}}=\mathbf{0 . 7 8 )}$ than with negative $(\bar{x}=0.88)$ emotions. There were no valence differences for LBDs or NCs. Finally, there was a main effect of Condition ( $F=19.68$; $\mathrm{df}=1.34 ; \mathrm{p}<$ 0.001 ), such that subjects displayed more appropriate expressions during the spontaneous $(\bar{x}=0.95)$ than during the posed $(\overline{\mathrm{x}}=0.81)$ condition.

Table 2 Mean scores (and standard deviations) for measures of emotional facial expression, by subject group

\begin{tabular}{lllll}
\hline & & & \\
\cline { 3 - 4 } Measure & Score range & $R B D s$ & $L B D s$ & NCs \\
\hline Responsivity* & $0-1$ & $0.59(0.19)$ & $0.74(0.14)$ & $0.72(0.14)$ \\
Appropriateness* & $0-1$ & $0.83(0.14)$ & $0.87(0.09)$ & $0.93(0.06)$ \\
Intensity & $1-7$ & $3.38(0.60)$ & $3.62(0.65)$ & $3.19(0.56)$ \\
\hline
\end{tabular}

*From impaired (low) to unimpaired (high).

†From minimal (low) to maximal (high). 
Intensity The three-way ANOVA conducted on the intensity scores yielded significant main effects for Condition $(F=9.66 ; \mathrm{df}=1.34 ; \mathrm{p}<0.005)$ and Valence $(F=6.11 ; \mathrm{df}=1.34 ; \mathrm{p}<0.05)$. Expressions were produced more intensely under posed $(\bar{x}=3.66)$ than spontaneous $(\overline{\mathrm{x}}=3 \cdot 12)$ conditions, and for positive $(\bar{x}=3.54)$ than negative $(\bar{x}=3.24)$ emotions. There were no significant main effects or interactions as a function of subject group. (See table 2 for group means.)

\section{Relationships between responsivity, appropriateness, and intensity}

To examine the relationships among the three measures of emotional facial expression, we intercorrelated the scores for responsivity, appropriateness, and intensity. Based on the ANOVA findings reported above for emotional expression, since there was only one Group difference as a function of condition or valence, data were collapsed across these distinctions into one mean score for each expression variable. Spearman rank-order correlation coefficients were computed among the three expression variables separately for each subject group and for all subjects. These correlation coefficients are displayed in table 3 . As can be seen in table 3, the only significant correlations were between responsivity and intensity. This was the case for both patient groups and for all subjects together. This finding suggests that the more responsive a subject was, the more intensely he expressed facial emotion.

\section{The relationship between emotional expression and non-emotional movement}

To examine the relationship between expression and movement, we correlated (Spearman rank-order) the scores for the three emotional facial expression variables (that is, responsivity, appropriateness, intensity) with scores for the six non-emotional facial movement variables: asymmetry of facial paralysis (upper, lower), adequacy of hemiface mobility (upper, lower), execution of praxis tasks, and accuracy of praxis tasks. This analysis was conducted only with the brain-damaged groups. For each expression variable, data were collapsed across condition and valence into one mean score. For hemiface mobility, the mean rating of the right and left hemifaces was used. RBDs and LBDs were treated separately. Among the 36 correlations computed (18 per patient group), there was only one significant $(p<0.05)$ correlation. (Since we were concerned that significant relationships might have been obscured by collapsing across the right hemiface and the left hemiface for the mobility ratings, the data were reanalysed with separate scores for each hemiface. Among the 24 correlations computed, 12 per patient group, only two were significant $p<$
Table 3 Spearman rank-order correlation rhos between parameters of facial emotional expression, by subject group.

\begin{tabular}{lccc}
\hline & \multicolumn{3}{l}{ Parameters } \\
\cline { 2 - 4 } $\begin{array}{l}\text { Subject } \\
\text { group }\end{array}$ & $\begin{array}{l}\text { Responsivity } \\
\text { vs appropriateness }\end{array}$ & $\begin{array}{l}\text { Responsivity } \\
\text { vs Intensity }\end{array}$ & $\begin{array}{l}\text { Intensity } \\
\text { vs appropriateness }\end{array}$ \\
\hline RBDs & 0.02 & $0.67^{*}$ & 0.37 \\
LBDs & 0.14 & $0.84 \dagger$ & -0.14 \\
NCs & -0.44 & 0.31 & -0.02 \\
All Ss & -0.06 & $0.63 \dagger$ & 0.01 \\
\hline
\end{tabular}

${ }^{*} \mathrm{p}<0.05$.

$+\mathrm{p}<0.001$.

0.05 , and no systematic patterns emerged.) The lack of significant correlations (median rho $=0.11$ ) suggests that the ability to make non-emotional facial movements is not related to the production of emotional facial expressions.

\section{Discussion}

The major purpose of this study was to assess systematically the effects of brain damge on the expression of facial emotion. It was hypothesised that patients with unilateral lesions of the right hemisphere would be more impaired on tasks of emotional facial expression than patients with lesions of the left hemisphere and normal controls. Examination of facial responsivity, appropriateness, and intensity suggested that patients with right hemisphere pathology, compared with patients with left hemisphere pathology or normal controls, were less responsive and less appropriate in their expression of facial emotion. For the appropriateness rating, this was only the case for the positive emotions. These findings were obtained for botho posed and spontaneous expressions. There were no? significant differences among the three groups in the intensity with which the facial expressions were produced.

The finding that RBDs were more impaired than LBDs and NCs in the expression of facial emotion lends support to the theory that the right hemisphere is dominant for emotional processing. ${ }^{39-41}$ While the bulk of evidence regarding the role of the right hemisphere in the facial expression of emotion has been based on studies examining facial asymmetry in normal subjects (for reviews, see ${ }^{124243}$ ), this study examines the emotional facial behaviour of subjects with verified lesions in either the right or left cerebral hemisphere.

When valence of emotional expression was considered, there were no systematic differences between positive and negative emotions across the three dependent variables. When the interaction between valence and subject group was examined, valence did not discriminate between patient groups either for responsivity or for intensity. However, RBDs were found to be significantly less appropriate than LBDs 
or NCs on positive emotions. This finding seems to converge with findings by Gardner and his colleagues $^{45}$ on the deficit of right hemisphere patients for the appreciation of humorous materials. In general, our finding of a selective deficit in RBDs for positive emotion contrasts with the literature on facial expression in normal controls which has reported evidence for right hemisphere mediation of negative emotion and left hemisphere or bilateral mediation of positive emotion (see review in ${ }^{1}$ ). It also contrasts with two previous clinical studies that also have considered this issue. ${ }^{1444}$ Sackeim and colleagues ${ }^{44}$ found evidence for right hemisphere mediation of negative emotions and left hemisphere mediation of positive emotion, while Buck and Duffy ${ }^{14}$ found no significant differences between RBDs and LBDs as a function of valence. Differences in patient populations (for example, aetiology, lesion location, subject characteristics) and in methodologies examining emotional behaviour (such as, elicitation conditons, stimuli, rating schemes) could account for these differences in results. Additional studies with larger numbers of subjects and more careful delineation of lesion location are necessary to assess more precisely the relationship between valence and lesion site.

When examining the relationships among the three parameters of emotional expression, there was a significant correlation between responsivity and intensity but no relation between appropriateness and either of these variables. The neuropsychological literature suggests the possibility that the relationship between responsivity and intensity reflects an underlying dimension such as arousal. In the current study, we used responsivity as an indirect measure of arousal. If one has an arousal deficit, it is possible that the amplitude or intensity of a behavioural response also may be dampened. The finding that responsivity was the only variable for which RBDs were consistenly imparied could be interpreted as support for a relationship between a deficit in arousal and some sort of right hemisphere dysfunction. There is a recent body of literature suggesting that arousal deficits are associated with the right hemisphere syndrome. For example, right hemisphere pathology has been associated with abnormal patterns of autonomic nervous system responding, as measured by heart rate ${ }^{46}$ and skin conductance, ${ }^{1847}$ and also with increases in reaction time. ${ }^{4849}$

When we examined the influence of non-emotional facial movement on the three aspects of facial emotional expression among the brain-damaged subject groups, correlations were nonsignificant. The braindamaged groups showed similar levels of performance on measures of both paralysis and mobility, indicating that group differences in facial mobility were not responsible for the lack of correlation. Al- though LBDs did demonstrate, as would be expected, significant impairments in bucco-facial apraxia, execution and accuracy measures of apraxia were not systematically related to our measures of facial emotional expression. These data, combined with data from related studies using normal subjects, ${ }^{1920}$ argue for the dissociation between systems controlling facial emotional expression and non-emotional facial movement.

\section{Conclusion}

In conclusion, we have observed deficits in the responsivity and appropriateness with which emotional facial expressions are produced as a function of right hemisphere pathology. These findings support the notion that the right hemisphere is dominant for certain aspects of facial emotional expression. The group differences for responsivity and the significant correlations between responsivity and intensity suggest that it may be fruitful for future research to examine the relationship between impairments in emotional facial expression and deficits in arousal among RBDs. Both patient groups had central facial paralysis and deficits in non-emotional muscular mobility on the hemiface contralateral to the lesion. The LBD group was apractic. It was not the case, however, that the ability to carry out specific facial expressions or to respond in emotional situations was affected by factors such as degree of facial paralysis, muscular facility, or bucco-facial apraxia.

This work was supported, in part, by USPHS Grant No MH37952 to New York University School of Medicine, USPHS Grant No NS06209 to Boston University School of Medicine, and Biomedical Research Support Grants RR07186-02 to Wellesley College and RR070604 to Queens College. We are grateful to Dr Ross Buck, Dr Mary Hyde, Jerry Martin, Karen Olsen, and the Boston VA Medical Center. This paper was presented, in part, at the International Neuropsychology Society Meeting, Denver, Colorado, February, 1986.

\section{References}

1 Borod JC, Koff E. Asymmetries in affective facial expression. In: Fox N, Davidson R, eds. The Psychobiology of Affective Development. Philadelphia: L Erlbaum and Associates, 1984.

2 Rinn WB. The neuropsychology of facial expression: A review of the neurological and psychological mechanisms for producing facial expression. Psychol Bull 1984;95:52-77.

3 Kuypers HGJM. Corticobulbar connexions to the pons and lower brain-stem in man-An anatomical study. Brain 1958;81:364-88.

4 Borod JC, Caron HS. Facedness and emotion related to lateral dominance, sex, and expression type. Neuropsychologia 1980;18:237-42.

5 Borod JC, Koff E, White B. Facial asymmetry in posed and spon- 
taneous expressions of emotion. Brain Cogn 1983;2:165-75.

6 Moscovitch M, Olds J. Asymmetries in spontaneous facial expression and their possible relation to hemispheric specialization. Neuropsychologia 1982;20:71-81.

7 Campbell R. Asymmetries in interpreting and expressing a posed facial expression. Cortex 1978;14:327-42.

8 Dopson WG, Beckwith BE, Tucker DM, Bullard-Bates PC. Asymmetry of facial expression in spontaneous emotion. Cortex 1984;20:243-52.

9 Heller W, Levy J. Perception and expression of emotion in righthanders and left-handers. Neuropsychologia 1981;19:363-72.

10 Sackeim HA, Gur RC. Lateral asymmetry in intensity of emotional expression. Neuropsychologia 1978;16:473-81.

11 Hager JC, Ekman P. The asymmetry of facial actions is inconsistent with models of hemispheric specialization. Psychophysiology 1985;22:307-318.

12 Borod JC, Koff E, Perlman Lorch M, Nicholas M. Channels of emotional expression in patients with unilateral brain damage. Arch Neurol 1985;42:345-8.

13 Borod JC, Koff E, Perlman Lorch M, Nicholas M. The expression and perception of facial emotion in brain-damaged patients. Neuropsychologia 1986;24:169-80.

14 Buck R, Duffy RJ. Nonverbal communication of affect in braindamaged patients. Cortex 1980;16:351-61.

15 Ross ED, Mesulam M. Dominant language functions of the right hemisphere? Prosody and emotional gesturing. Arch Neurol 1979;36:144-8.

16 Gardner $H$. The shattered mind: The person after brain damage. New York: Alfred A Knopf, 1975.

17 Gainotti G. Emotional behavior and hemispheric side of the lesion. Cortex 1972;8:41-55.

18 Heilman KM, Schwartz H, Watson RT. Hypoarousal in patients with the neglect syndrome and emotional indifference. Neurology 1978;28:229-32.

19 Borod JC, Koff E. Hemiface mobility and facial expression asymmetry. Cortex 1983;19:355-61.

20 Sackeim HA, Gur RC. Asymmetry in the face. Science 1980;209:834-6.

21 Koff E, Borod JC, White B. Asymmetries for hemiface size and mobility. Neuropsychologia 1981;19:825-30.

22 Gordon AS, Friedberg J. Current status of testing for seventh nerve lesions. Otolaryngol Clin North Am 1978;11:301-24.

23 Goodglass H, Kaplan E. The Assessment of Aphasia and Related Disorders. Philadelphia: Lea and Febiger, 1972.

24 Hollingshead AB, Redlich FC. Social Class and Mental Illness. New York: Wiley, 1958.

25 Wechsler D. The Measurement and Appraisal of Adult Intelligence. Baltimore: Williams and Wilkins, 1958.

26 Borod JC, Koff E, Perlman Lorch M, Nicholas M. Deficits in facial expression and movement as a function of brain damage. In: Nespoulous JL, Perron P, Le Cours AR, eds. The Biological Foundations of Gestures: Motor and Semiotic aspects. Hillsdale NJ: L Erlbaum Associates, 1986.

27 Chusid JG, McDonald JF. Correlative Neuroanatomy and Functional Neurology (17th ed.). Los Altos CA: Lange Medical Publications, 1976.

28 De Jong RN. The Neurologic Examination (4th ed.). Hagerstown MD: Harper \& Row, 1979.
29 Diamond C, Frew I. The Facial Nerve. New York: Oxford University Press, 1979.

30 Devriese PP. Experiments on the Facial Nerve. Amsterdam: North-Holland Publishing Company, 1972.

31 Miehlke A. Surgery of the Facial Nerve. Philadelphia PA: WB Saunders, 1973.

32 Van Gelder R. Facing the Facial Nerve. Unpublished manuscript. Amsterdam: Interfaculteit Lichamelijke Opvoeding, Vrjie Universiteit, 1981

33 Albert ML, Goodglass H, Helm NA, Rubens AB, Alexander MP. Clinical Aspects of Dysphasia. New York: Springer-Verlag, 1981:49.

34 Buck $R$. The slide viewing technique for measuring non-verbal sending accuracy: a guide for replication. Catal Sel Doc Psychol 1978;8:62.

35 Ekman P, Friesen W. Unmasking the Face. Englewood Cliffs NJ: Prentice-Hall, 1975.

36 Izard CD. The Face of Emotion. New York: Appleton-CenturyCrofts, 1971.

37 Campbell R. Asymmetries in moving faces. $\mathrm{Br} \mathrm{J}$ Psychol 1982;73:95-103.

38 Winer BJ. Statistical Principles in Experimental Design. New York: McGraw-Hill Book Company, 1971.

39 Borod JC, Koff E, Caron H. Right hemispheric specialization for the expression and appreciation of emotion: A focus on the face. In: Perecman E, ed. Cognitive Processes in the Right Hemisphere. New York: Academic Press, 1983.

40 Ley RG, Bryden MP. Consciousness, emotion, and the right hemisphere. In: Stevens R, Underwood G, eds. Aspects of Consciousness. New York: Academic Press, 1982.

41 Tucker DM. Lateral brain function, emotion, and conceptualization. Psychol Bull 1981;89:19-46.

42 Hager JC. Asymmetries in facial expression. In: Ekman P, ed. Emotion in the Face: Guidelines for Research and an Integration of Findings. Cambridge: Cambridge University Press, 1982.

43 Sackeim HA, Gur RC. Facial asymmetry and the communication of emotion. In: Cacioppo JT, Petty RE, eds. Social Psychophysiology. New York: Guilford Press, 1982.

44 Sackeim H, Greenberg M, Weiman A, Gur R, Hungerbuhler J, 응 Geshwind N. Functional brain asymmetry in the expression of positive and negative emotions: Lateralization of insult in cases of uncontrollable emotional outbursts. Arch Neurol 1982;19:210-8.

45 Bihrle AM, Brownell HH, Powelson JA, Gardner H. Comprehension of humorous and nonhumorous materials by left and right brain-damaged patients. Brain Cogn 1986;5:399-411.

46 Boller F, Yokoyama K, Ackles P, Hood P, Jennings R. Lack of heart rate changes during an attention task in patients with right hemisphere lesions. Neurology 1986;36 (Suppl 1):131.

47 Morrow L, Vrtunski B, Kim Y, Boller F. Arousal responses to emotional stimuli and laterality of lesion. Neuropsychologia 1981;19:65-71.

48 DeRenzi E, Faglioni P. The comparative efficiency of intelligence and vigilance in detecting hemisphere damage. Cortex 1965;1:410-33.

49 Howes D, Boller F. Simple reaction times: Evidence for focal impairment from lesions of the right hemisphere. Brain 1975;98:317-32. 